\title{
Motivos para a prática de exercício físico em universitários e fatores associados
}

CDD. 20.ed. 159

613.7

\author{
Dartagnan Pinto GUEDES \\ Rosimeide Francisco Santos LEGNANI** \\ Elto LEGNANI ${ }^{* * * *}$
}

*Faculdade de Educação Física, Universidade Norte do Paraná. **Universidade Estadual de Ponta Grossa. ***Universidade Tecnológica Federal do Paraná.

\section{Resumo}

Objetivo do estudo foi identificar motivos para prática de exercício físico em universitários de acordo com sexo, idade e condição econômica. Amostra foi constituida por 2380 sujeitos (1213 moças e 1167 rapazes), com idades entre 18 e 35 anos. Motivos para prática de exercício físico foram identificados mediante versão traduzida do Exercise Motivations Inventory (EMI-2). Rapazes reportaram oferecer significativamente maior importância para prática de exercício físico em motivos identificados com Condição Física e Competição, e moças com Controle de Peso Corporal e Aparência Física. Motivos relacionados à Prevenção de Doenças, ao Controle de Peso Corporal e à Reabilitação de Saúde aumentaram significativamente com a idade. Motivos associados ao Controle de Peso Corporal e à Aparência Física receberam importância significativamente maior pelos universitários mais privilegiados economicamente. Concluindo, resultados encontrados poderão contribuir para delineamento de programas de promoção de exercício físico e provável redução dos casos de abandono entre os universitários.

UnIteRMos: Motivação; EMI-2; Atividade física; Adulto jovem.

\section{Introdução}

Um dos temas que tem suscitado grande interesse no cenário da atividade física relacionada à saúde refere-se aos motivos que levam o indivíduo a iniciar um programa de exercício físico e às razões que justificam sua permanência por algum tempo. Neste caso, os motivos que eventualmente possam estar associados à prática de exercício físico são bastante variados (WEInBERD \& Gould, 2011).

Embora possa ser possível identificar motivos comuns nos estudos disponibilizados, verifica-se que estes se modificam em função da interação entre diferentes atributos demográficos e situações específicas (ANDRAde Bastos, Salguero, Gonzales-Boto \& Marquez, 2006; Dacey, Baltzell \& ZaichKowsKy, 2008; EBBEN \& BRUDZYNSKI, 2008; INGLEDEW \& MARKLAND, 2008; Keele, 2009; Kilpatrick, Hebert \& Bartholomew, 2005), destacando-se a importância da realização de estudos com essa finalidade. A identificação dos motivos subjacentes à prática de exercício físico permite que sejam delineadas ações que possam levar o praticante a alcançar em sua maior plenitude as metas propostas, promovendo, desse modo, clima motivacional favorável, o que eleva as chances de aderência aos programas e, por consequência, reduz a possibilidade de abandono (Anderson, 2003; EbBen \& Brudzynski, 2008).

Claramente, sexo e idade se destacam como atributos importantes na definição dos motivos associados à pratica de exercício físico (ANDRADE BASTOS et al., 2006; BRUnet \& SABISTON, 2011; Kilpatrick, Hebert \& Bartholomew, 2005; Quindry, Yount, O'Bryant \& Rudisill, 2011). Contudo, atributos sociais e ambientais, além do contexto cultural em que se esta inserido, deverá modular a seleção desses motivos (Cagas, Torre \& Manalastas, 2010; Chen, 1998; Keele, 2009; Withall, Jago \& Fox, 2011).

Além do que, os motivos para a prática de exercício físico podem apresentar especificidade de acordo com o segmento populacional considerado. Neste caso, raros foram os estudos realizados envolvendo o segmento universitário e, mais escassos ainda, são os estudos envolvendo estudantes universitários brasileiros. De destacar a importância de estudos envolvendo 
populaçôes universitárias, considerando que, em idades de acesso e permanência nas universidades, o risco de incorporar hábitos sedentários se eleva acentuadamente, em razão da necessidade de se dedicar muitas horas do dia aos estudos com consequente redução do tempo dedicado ao lazer ativo fisicamente (CALFAS, Sallis, Lovato \& Campbell, 1994; Keating, Guan, PiÑERO \& BRIDGES, 2005). Portanto, experiências de prática de exercício físico no ambiente universitário pode se converter em satisfatório preditor de prática futura em idades adultas mais avançadas.

Evidências de que a prática de exercício físico guarda relação significativa e positiva com benefícios associados à saúde individual em diferentes períodos etários, têm sido reconhecidas por inúmeros estudos disponibilizados na literatura (BAUMAN, 2004; Nocon, Hiemann, Müller-Riemenschneider, Thalau, Roll, Willich, 2008; Warburton, Nicol \& Bredin,

\section{Método}

A população de referência para o estudo incluiu estudantes dos 42 cursos de graduação da Universidade Estadual de Londrina. Esta Instituição pertence à Rede Pública de Ensino Universitário do Estado do Paraná e sua comunidade estudantil é formada por aproximadamente 15 mil estudantes universitários. Os procedimentos de seleção da amostra obedeceram a uma sequência de etapas para se obter uma amostragem probabilística por conglomerados, tendo como referência o número de estudantes universitários quanto ao sexo, ao curso, à área de estudo e ao turno que frequentava as aulas (diurno, noturno e período integral).

Neste caso, dos 2380 estudantes universitários selecionados na amostra (1213 moças e 1167 rapazes), $45 \%$ deles apresentavam idades $\leq 20$ anos, $48 \%$ entre 21 e 29 anos e $7 \%$ restante $\geq 30$ anos. Com relação à classe econômica familiar, $51,4 \%$ foram categorizados nas classes A e B (maior nível econômico), 15,1\% nas classes $\mathrm{D}$ e $\mathrm{E}$ (menor nível econômico) e os demais 33,5\% na classe C (nível econômico intermediário). Ainda, no que se refere aos cursos de formação, proporçôes similares próximas de $25 \%$ de estudantes universitários selecionados para o estudo se distribuíram nas quatro áreas de estudo: humanas, jurídicas/sociais, exatas/tecnológicas e biológica/saúde.

As informaçôes relacionadas aos motivos para a prática de exercício físico foram obtidas mediante a aplicação do Exercise Motivations Inventory (EMI-2), traduzido, adaptado e validado para utilização na população jovem
2006). Em vista disso, órgãos e instituições vinculadas à saúde pública têm procurado incentivar a sua prática (Haskell, Lee, Pate, Powell, Blair, Franklin, MaCera, Heath, Thompson \& Bauman, 2007; WHO, 2010). Porém, curiosamente, tem sido identificado, não somente aumento na proporção de sujeitos que ingressam em programas de exercício físico, mas também, e sobretudo, os casos de abandono (ANDERSON, 2003; EbBen \& BRUdzynski, 2008). Consequentemente, se faz necessário demarcar e conhecer os processos motivacionais que possam levar o sujeito a praticar exercício físico, supondo que, dessa forma, torna-se possível compreender e intervir na prevenção de possível abandono.

O objetivo do estudo foi identificar os motivos para a prática de exercício físico em amostra representativa de universitários em função de sexo, idade e classe econômica familiar.

brasileira (Guedes, LegnANi \& LeGNANI, 2011), com questôes adicionais relacionadas ao sexo, à idade e aos indicadores direcionados à determinação da classe econômica familiar. Os procedimentos para definição da classe econômica familiar acompanharam diretrizes propostas pela AssociaÇÃo NaCiOnAL de EMPRESAS DE PESQUISA - ABEP (2008), envolvendo informações relatadas quanto à posse de utensílios domésticos e grau de instrução de membros da família.

O EMI-2, originalmente idealizado em língua inglesa (MarKLAND \& INGLEDEW, 1997), é um dos instrumentos mais frequentemente utilizado para identificar os motivos associados à prática de exercício físico e tem permitido estabelecer comparações mais seguras e confiáveis entre os estudos (CAGAS, TORRE \& Manalastas, 2010; Capdevila, Niñerola \& Pintanel, 2004; Ingledew, MarkLand \& Ferguson, 2009; INGLEDEW \& Sullivan, 2002; Loze \& Collins, 1998; MaltBy \& DAY, 2001). A versão traduzida do EMI-2 é constituída por 44 itens, agrupados em 10 fatores de motivação, representando amplo espectro de motivos para a prática de exercício físico definidos "a priori" e validados mediante recursos da análise fatorial confirmatória: diversão/bem-estar, controle de estresse, reconhecimento social, afiliação, competição, reabilitação de saúde, prevençâa de doenças, controle de peso corporal, aparência física e condição fisisca. Em seu delineamento o indivíduo se posiciona frente aos 44 itens que compõe o inventário, mediante escala 
Lickert de seis pontos $(0=$ "nada verdadeiro" a $5=$ "totalmente verdadeiro"), encabeçado pelo enunciado "Pessoalmente, Eu pratico (ou poderia vir a praticar) exercício físico ....”. De acordo com seus idealizados e acompanhando a teoria de autodeterminação (DECI \& RYAN, 1985), o EMI-2 permite identificar, dimensionar e ordenar fatores de motivação intrínseca e extrínseca para a prática de exercício físico.

$\mathrm{O}$ instrumento de medida foi aplicado em um único momento, individualmente para cada estudante universitário e por um mesmo pesquisador. Para tanto, as salas de aula sorteadas para estudo foram visitadas pelo pesquisador e os estudantes universitários foram esclarecidos quanto aos objetivos do estudo, princípios de sigilo e de não influência no desempenho acadêmico. Aqueles estudantes universitários que assinaram o Termo de Consentimento Livre e Esclarecido receberam cópia do EMI-2 com instruçôes para o seu autopreenchimento e informação quanto à disposição do pesquisador para eventuais esclarecimentos. Após o preenchimento e a devolução do instrumento preenchido, este

\section{Resultados}

A TABELA 1 apresenta valores equivalentes aos fatores de motivação para prática de exercício físico apontado pelos universitários. Ao considerar a hierarquia dos valores médios encontrados para cada fator de motivação, verifica-se que o fator significativamente de maior destaque foi característico de um contexto de motivação relacionada à Prevenção de Doenças $(3,49 \pm 0,61)$. Os fatores de menor destaque em linguagem estatística se identificaram em um contexto de motivação relacionada ao Reconhecimento Social $(1,23 \pm 0,39)$ e à Competição $(1,61 \pm 0,61)$. Os demais fatores de motivação foram reunidos em posição intermediária em que os valores médios dos fatores Condição Física $(2,95 \pm 0,69)$, Controle de Peso Corporal $(2,90 \pm 0,85)$, Aparência Física $(2,80 \pm 0,68)$ e Controle de Estresse $(2,80 \pm 0,77)$ se diferiram significativamente foi armazenado em uma urna juntamente com os demais. Os dados foram coletados entre os meses de maio e junho de 2008.

O tratamento estatístico dos dados foi realizado por intermédio do pacote computadorizado "Statistical Package for the Social Science" (SPSS) - versão 17.0. Inicialmente foi testada a distribuição de frequência por intermédio do teste de Kolmogorov-Smirnov. Considerando que os dados mostraram distribuição de frequência normal, recorreu-se aos recursos da estatística paramétrica, mediante cálculo de média e desviopadrão. Posteriormente, para estabelecer comparações entre os estratos formados, foram utilizadas análises de variância multivariada (MANOVA) e univariada (ANOVA), tendo como variáveis dependentes os fatores de motivação e variáveis independentes o sexo, a idade e a classe econômica familiar, acompanhadas do teste de comparação múltipla de Scheffe para identificação de diferenças específicas. $\mathrm{O}$ delineamento do estudo foi aprovado pelo Comitê de Ética em Pesquisa Envolvendo Seres Humanos da Universidade Estadual de Londrina, Paraná (Parecer 214/07).

dos valores médios encontrados nos fatores Diversãol Bem-Estar (2,40 \pm 0,70), Afiliação $(2,28 \pm 0,66)$ e Reabilitação de Saúde $(2,16 \pm 0,74)$.

Ao assumir o ponto-de-corte arbitrário equivalente a " 2,5 " na escala de medida das respostas do EMI-2, o que corresponde a uma referência equidistante entre os escores extremos da escala de medida (opções de escores entre zero e cinco), constatase que cinco fatores motivacionais apresentaram valores médios acima deste patamar (Prevenção de Doenças, Condição Física, Controle de Peso Corporal, Aparência Física e Controle de Estresse), enquanto os outros cinco fatores motivacionais considerados apresentaram valores médios abaixo deste patamar (Reconhecimento Social, Competição, Reabilitação de Saúde, Afiliação e Diversão/Bem-Estar). 
GUEDES, D.P.; LEGNANI, R.F.S. \& LEGNANI, E.

TABELA 1 - Informações estatísticas (média \pm desvio-padrão) dos fatores de motivação para a prática de exercício físico apontados por universitários mediante aplicação do EMI-2.

Valores subscritos pelas mesmas letras indicaram similaridades estatísticas $(p<0,01)$.

\begin{tabular}{lcc}
\hline Fatores & Média \pm Desvio-Padrão & \\
\hline Prevenção de doenças & $3,49 \pm 0,61^{\mathrm{a}}$ & Fatores \\
Condição física & $2,95 \pm 0,69^{\mathrm{b}}$ & mais \\
Controle de peso corporal & $2,90 \pm 0,85^{\mathrm{b}}$ & \\
Apparência física & $2,80 \pm 0,68^{\mathrm{b}}$ & \\
Controle de estresse & $2,80 \pm 0,77^{\mathrm{b}}$ & Fatores \\
\hline Diversão/Bem-estar & $2,40 \pm 0,70^{\mathrm{c}}$ & menos \\
Afiliação & $2,28 \pm 0,66^{\mathrm{c}}$ & importantes \\
Reabilitação da saúde & $2,16 \pm 0,74^{\mathrm{c}}$ & \\
Competição & $1,61 \pm 0,61^{\mathrm{d}}$ & $1,23 \pm 0,39^{\mathrm{d}}$ \\
Reconhecimento social & & \\
\hline
\end{tabular}

Indicadores estatísticos encontrados mediante realização da MANOVA revelaram efeito significativo de sexo (Wilks' Lambda = 0,737; F (10,2369) = 84,736; $\mathrm{p}<0,001$ ), idade (Wilks' Lambda = 0,958; $\mathrm{F}(30,8973)=2,560 ; \mathrm{p}<0,001)$ e classe econômica familiar (Wilks' Lambda $=0,957 ; \mathrm{F}(30,8973)=$ 2,632; $\mathrm{p}<0,001)$ no grau de importância apontado pelos universitários para a prática de exercício físico.

A TABELA 2 apresenta informações estatísticas equivalentes aos fatores de motivação separadamente por sexo. Os resultados da análise de covariância, controlando estatisticamente a idade e a classe econômica familiar dos universitários, apontaram diferenças estatísticas entre ambos os sexos em quatro fatores de motivação considerados. As moças apresentaram valores médios significativamente mais elevados nos fatores Controle de Peso Corporal $(\mathrm{F}=8,437 ; \mathrm{p}<0,001)$ e Aparência Física $(\mathrm{F}=2,896$; $\mathrm{p}=0,046)$. Enquanto os rapazes demonstraram valores médios significativamente mais elevados nos fatores Competição $(\mathrm{F}=10,414 ; \mathrm{p}<0,001)$ e Condição Física $(\mathrm{F}=6,846 ; \mathrm{p}<0,001)$. No que se refere à organização hierárquica, tendo como referência a magnitude dos valores médios de cada fator de motivação, entre aqueles fatores apontados como os de menor importância, as diferenças observadas na disposição hierárquica foram bastante discretas. Contudo, apesar do fator Prevenção de Doenças ter sido apontado igualmente como o fator mais importante pelos universitários de ambos os sexos (moças: $3,65 \pm 0,59$; rapazes: $3,32 \pm 0,61$ ), as moças apontaram o Controle de Peso Corporal como o segundo fator motivacional mais importante $(3,30$ $\pm 0,83$ ), enquanto os rapazes apontaram esse mesmo fator motivacional tão somente como o sexto fator mais importante $(2,49 \pm 0,81)$. Em contrapartida, os rapazes apontaram a Condição Física como o segundo fator motivacional mais importante $(3,24$ $\pm 0,66)$, sendo que as moças apontaram esse fator como de importância intermediária $(2,67 \pm 0,69)$. 
TABELA 2 - Organização hierárquica $(\mathrm{OH})$, média (Md), desvio-padrão (Dp) e estatística $\mathrm{F}$ dos fatores de motivação para a prática de exercício físico de acordo com o sexo dos universitários.

\begin{tabular}{|c|c|c|c|c|c|c|}
\hline \multirow{3}{*}{$\begin{array}{l}\text { Fatores } \\
\text { Prevenção de Doenças }\end{array}$} & \multicolumn{2}{|c|}{ Moças } & \multicolumn{2}{|c|}{ Rapazes } & \multirow{2}{*}{\multicolumn{2}{|c|}{ Teste F }} \\
\hline & \multirow{2}{*}{$\begin{array}{c}\mathbf{O H} \\
1\end{array}$} & \multirow{2}{*}{$\frac{\mathbf{M d} \pm \mathbf{D} \mathbf{p}}{3,65 \pm 0,59}$} & \multirow{2}{*}{$\begin{array}{c}\mathbf{O H} \\
1\end{array}$} & \multirow{2}{*}{$\frac{\mathbf{M d} \pm \mathbf{D} \mathbf{p}}{3,32 \pm 0,61}$} & & \\
\hline & & & & & 1,156 & ns \\
\hline Condição Física & 5 & $2,67 \pm 0,69$ & 2 & $3,24 \pm 0,66$ & 6,846 & $<0,001$ \\
\hline Controle de Peso Corporal & 2 & $3,30 \pm 0,83$ & 6 & $2,49 \pm 0,81$ & 8,437 & $<0,001$ \\
\hline Aparência Física & 3 & $2,98 \pm 0,68$ & 4 & $2,58 \pm 0,65$ & 2,896 & 0,046 \\
\hline Controle de Estresse & 4 & $2,95 \pm 0,76$ & 3 & $2,64 \pm 0,76$ & 2,116 & ns \\
\hline Diversão/Bem-Estar & 6 & $2,56 \pm 0,70$ & 7 & $2,28 \pm 0,70$ & 1,811 & ns \\
\hline Afiliação & 8 & $2,14 \pm 0,62$ & 5 & $2,53 \pm 0,67$ & 2,213 & ns \\
\hline Reabilitação da Saúde & 7 & $2,35 \pm 0,76$ & 9 & $1,90 \pm 0,69$ & 2,351 & ns \\
\hline Competição & 10 & $1,18 \pm 0,49$ & 8 & $2,10 \pm 0,65$ & 10,414 & $<0,001$ \\
\hline Reconhecimento Social & 9 & $1,02 \pm 0,40$ & 10 & $1,43 \pm 0,55$ & 2,398 & ns \\
\hline
\end{tabular}

Análise de covariância mediante controle das informações associadas à idade e à classe econômica familiar.
As informações estatísticas equivalentes aos fatores de motivação tendo em conta a idade dos universitários são disponibilizadas na TABELA 3. Os resultados da análise de covariância, mediante controle estatístico quanto ao sexo e à classe econômica familiar dos universitários, apontaram diferenças significativas em quatro dos fatores motivacionais considerados. $\mathrm{O}$ fator de motivação relacionado à Competição $(\mathrm{F}=5,154 ; \mathrm{p}<0,001)$ apresentou valores médios significativamente menores com o avanço da idade, ao passo que os fatores de motivação vinculados à Reabilitação de Saúde (F = 4,166; $\mathrm{p}=0,002)$, à Prevenção de Doenças $(\mathrm{F}=$ 8,250; $\mathrm{p}<0,001$ ) e ao Controle de Peso Corporal (F $=2,511 ; \mathrm{p}=0,040)$ demonstraram valores médios mais elevados em linguagem estatística ao longo da idade. A organização hierárquica associada à magnitude dos valores médios de cada fator de motivação foi bastante semelhante nos grupos etários definidos, sugerindo, desse modo, que a ordem de importância dos fatores de motivação para a prática de exercício físico tendeu a não se alterar com a idade dos universitários.

Quanto à classe econômica familiar, na TABELA 4 são observadas as informações estatísticas associadas à análise de covariância mediante controle de sexo e idade dos universitários. Os resultados sugerem que os valores médios dos fatores de motivação tenderam a ser mais elevados nas classes econômicas familiar mais altas. Contudo, as diferenças encontradas foram detectadas estatisticamente somente no caso dos fatores de motivação relacionados ao Controle de Peso Corporal $(\mathrm{F}=3,849 ; \mathrm{p}=0,004)$ e à Aparência Física $(\mathrm{F}=5,011 ;$ $\mathrm{p}=0,001)$, e entre os valores médios identificados nos universitários pertencentes à classe econômica familiar mais alta (Classe A) em comparação com os universitários pertencentes à classe econômica familiar mais baixa (Classe D e E). Por outro lado, apesar das diferenças significativas observadas entre os valores médios, constata-se que a organização hierárquica dos fatores de motivação foi bastante semelhante nos quatro estratos econômicos considerados. 

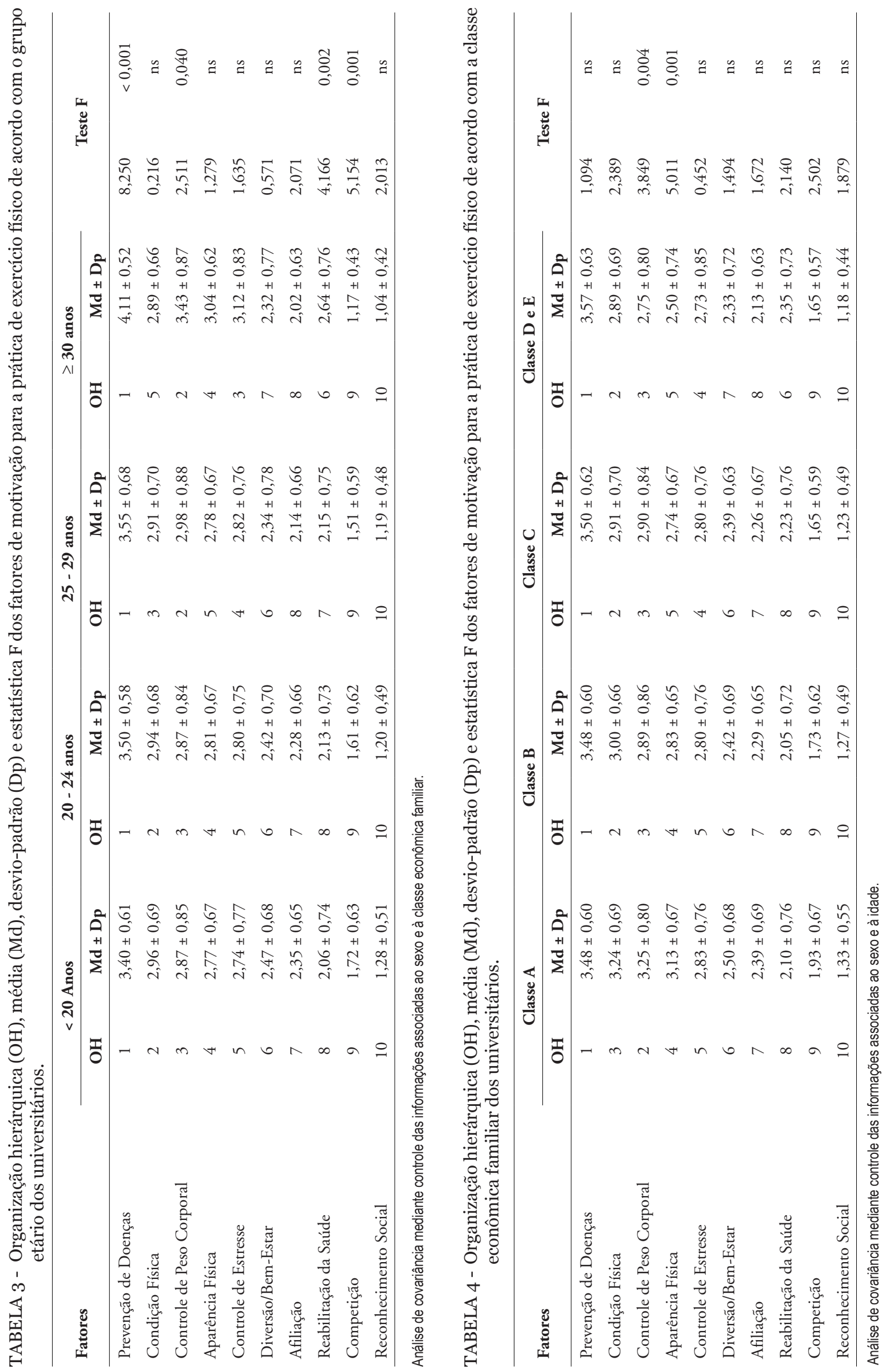

684 • Rev. bras. Educ. Fís. Esporte, São Paulo, v.26, n.4, p.679-89, out./dez. 2012 


\section{Discussão}

A motivação para a prática de exercício físico é uma característica psicológica multidimensional influenciada por aspectos intrínsecos do sujeito, como é o caso de preferências, desejos, temores, entre outros, e por experiências extrínsecas em seu entorno, como aceitação social, amizade, habilidades e competências, entre outros (WeInBERG \& GOULD, 2011). No momento em que vem sendo incentivada a adoção de hábitos mais ativos fisicamente em todos os segmentos da população (HASKELL et al., 2007; WHO, 2010), é fundamental ter presente os principais motivos que levam grupos populacionais específicos a praticarem exercício físico, na tentativa de contribuir para sua adesão e minimizar a possibilidade de abandono. O presente estudo procurou identificar fatores de motivação relacionados à prática de exercício físico em amostra representativa de universitários. Esta aproximação permite conhecer as características motivacionais de diferentes estratos de praticantes e potenciais praticantes de exercício físico, disponibilizando informaçōes relevantes que favoreçam intervenções mais efetivas com propósito de fomentar a prática de exercício físico no âmbito universitário.

De maneira geral, Prevenção de Doenças foi o principal fator de motivação para a prática de exercício físico apontado pelos universitários. Na sequência, os outros fatores assinalados pelos universitários que mais motivam, ou que poderiam motivá-los, para a prática de exercício físico também foram vinculados à motivação extrínseca: Condição Física, Controle de Peso Corporal, Aparência Física e Controle de Estresse. Achado de destaque é o fato dos fatores Diversãol Bem-Estar e Afiliação, dois importantes fatores associados à motivação intrínseca, terem sido igualmente contemplados em posição significativamente inferior diante dos fatores classicamente associados à motivação extrínseca e similar ao fator Reabilitação de Saúde. Os fatores vinculados à Competição e ao Reconhecimento Social se posicionaram como atributos de menor grau de motivação relatados pelos universitários.

Apesar de serem identificadas diferenças quanto à magnitude dos valores médios, a organização hierárquica dos fatores de motivação identificada nos universitários aqui analisados e na amostra de sujeitos britânicos reunida no estudo original de proposição do EMI-2 (MARKLAND \& INGLEDEW, 1997) é bastante similar. Esses resultados corroboram com achados apresentados por outros estudos, envolvendo diferentes escalas de medida, que apontam cuidados preventivos com a saúde e preocupação com a condição física, o peso corporal e a aparência física como dimensōes que mais motivam adultos, universitários ou não universitários, a se manterem ativos fisicamente mediante a prática de exercício físico (ANDRADE BASTOS et al., 2006; Brunet \& Sabiston, 2011; Cagas, Torre \& Manalastas, 2010; Cash, Novy \& Grant, 2004; Chen, 1998; Ingledew \& MarkLand, 2008; Kilpatrick, Hebert \& Bartholonew, 2005; Maltby \& DaY, 2001; NeTZ \& RaVIV, 2004).

Ao relacionar os motivos para a prática de exercício físico com o sexo dos universitários, constatou-se que as moças atribuíram grau de importância ao Controle de Peso Corporal e à Aparência Física significativamente mais elevado; enquanto os rapazes valorizaram em maior grau os motivos relacionados à Condição Física e à Competição. Evidências disponibilizadas na literatura confirmam a tendência do sexo feminino em se identificar mais intensamente com motivos estéticos para a prática de exercício físico, ao contrário do sexo masculino que tende a valorizar atributos relacionados ao desafio e à competência pessoal (ANDRADE BASTOS et al., 2006; BRUNET $\&$ Sabiston, 2011; Cagas, Torre \& Manalastas, 2010; Kilpatrick, Hebert \& Bartholonew, 2005; WiLSON \& RodGers, 2002). Esses resultados trata-se de um achado interessante, na medida em que sugere a ocorrência de similaridades no grau de importância apontado pelas moças e pelos rapazes nos atributos vinculados à motivação intrínseca, e a existência de diferenças significativas entre ambos os sexos nos atributos vinculados à motivação extrínseca.

Quanto à idade, apesar dos valores médios observados no fator de motivação Competição serem os mais baixos comparativamente com os demais fatores, universitários com $<20$ anos atribuíram importância significativamente mais elevada a este fator que universitários com $\geq 30$ anos, o que confirma a pré-disposição típica dos mais jovens em enfrentar desafios e colocar a prova sua competência pessoal (Yan \& McCullagh, 2004). Aqueles universitários com idades $\geq 30$ anos relataram ser significativamente mais motivados que seus pares mais jovens para a prática de exercício físico por conta de atributos vinculados à Prevenção de Doenças, ao Controle de Peso Corporal e à Reabilitação de Saúde. Resultados semelhantes foram identificados em estudos anteriores (ANDRAde Bastos et al., 2006; BRUNet \& SABISTON, 2011; NeTZ \& RaVIV, 2004; QUINDRY et al., 2011) e podem ser justificados em razão da preocupação com a saúde inerente ao avanço da idade. 
Em tese, o modelo teórico de adesão à prática de exercício físico relacionado à crença na saúde pode oferecer explicação plausível para as diferenças significativas observadas neste contexto. Neste caso, mesmo considerando que as agressōes para a saúde induzidas pelo sedentarismo possam estar presentes desde as idades mais precoces, somente com a percepção de ameaça e suscetibilidade às doenças, que ocorre a partir de idades mais avançadas, é que os sujeitos tendem a adotar concepçôes de promoção da saúde e prevenção/reabilitação de doenças com relação à prática de exercício físico.

Estudos anteriores também apontaram evidências favoráveis quanto à eventual relação entre classe econômica e fatores de motivação para a prática de exercício físico (Withall, JaGOR \& Fox, 2011). No presente estudo, aqueles universitários pertencentes à classe econômica familiar mais elevada (Classe A) demonstraram ser significativamente mais motivados para a prática de exercício físico em comparação com universitários pertencentes à classe econômica familiar menos privilegiada (Classe D e E) por intermédio dos fatores Controle de Peso Corporale Aparência Física. Neste caso, coincidindo com resultados encontrados em estudos envolvendo adolescentes (InGLEDEW \& Sullivan, 2002) assume-se que, entre universitários de mais elevado nível econômico familiar, em razão do contexto sociocultural em que estão inseridos, possa existir maior preocupação com motivos estéticos relacionados à prática de exercício físico, levando-os a priorizar a busca por um corpo magro e por uma imagem corporal que atenda aos padrões de beleza impostos pela sociedade atual.

Os achados do estudo podem ser interpretados a luz da teoria de autodeterminação (DECI \& RYAN, 1985; Hagger \& Chatzisarantis, 2008). Neste caso, quando motivados intrinsecamente os praticantes tendem a se envolver com o exercício físico aparentemente pelo prazer e satisfação inerentes que derivam de sua própria prática, sendo que, de fato, esta prática atende necessidades psicológicas de autonomia, competência e autorealização. Quando extrinsecamente motivados os sujeitos procuram se envolver com o exercício físico fundamentalmente para satisfazer demandas impostas externamente ou para obter recompensas que são atribuídas através de sua prática. Portanto, diferentes orientaçôes motivacionais podem apresentar diferentes consequências cognitivas, emocionais e comportamentais.

Acompanhamento longitudinal tem mostrado que, motivos identificados em dimensões intrínsecas, mas não em dimensões extrínsecas, são mais efetivos e susceptíveis de serem sustentados por mais longo tempo (VIERling, Standage \& TREASURE, 2007). Logo, sujeitos motivados intrinsecamente para o exercício físico deverão apresentar maior possibilidade de aderência a sua prática que sujeitos motivados mediante dimensões externas. Ainda, observaçōes experimentais constataram que muitos sujeitos iniciam a prática de exercício físico por questões de saúde e redução do peso corporal; porém, na sequência, poucos desses sujeitos permanecem se exercitando regularmente a menos que encontrem prazer e satisfação em sua prática (WeInberg \& Gould, 2011).

O presente estudo apresenta algumas limitações que devem ser consideradas. Neste caso, é importante referir que as informações equivalentes aos fatores de motivação para a prática de exercício foram auto-relatadas. No entanto, auto-relato é um procedimento corrente em estudos com essas características, sendo a forma mais viável de realizar levantamentos em larga escala. Por outro lado, o maior tamanho amostral permite, de alguma forma, minimizar eventual imprecisão das estimativas calculadas. Também, a abordagem transversal dos dados pode limitar o estabelecimento de associações sem que seja equacionada a possibilidade de existir causalidade inversa.

Concluindo, evidências encontradas no presente estudo apontaram diferenças específicas em fatores de motivação para a prática de exercício físico de acordo com sexo, idade e classe econômica familiar. Contudo, importante resultado a ser destacado refere-se à tendência dos universitários em identificar fatores relacionados às dimensões externa de motivação (Prevenção de Doenças, Condição Física, Controle de Peso Corporal, Aparência Física $e$ Controle de Estresse) como agentes motivadores cruciais para a prática de exercício físico. Neste sentido, baseando-se nos pressupostos da teoria de autodeterminação, os achados sugerem que os universitários analisados no estudo, por priorizarem fatores associados à motivação extrínseca, deverão apresentar maior dificuldade de estabelecerem uma aderência mais efetiva e duradoura voltada à prática de exercício físico.

Particularmente, os resultados observados no estudo podem ser úteis para profissionais que atuam no campo da educação física e da saúde pública, por oferecer subsídios que venham auxiliar no delineamento de programas de exercício físico no âmbito universitário, mediante açôes que possam potencializar o grau de motivação de acordo com as dimensões que compõe o $E M I-2$. 


\begin{abstract}
Exercise motives in college students and associated factors

Purpose of this study was to identify the exercise motives in college students according to gender, age and economic status. A total of 2380 subjects ( 1213 females and 1167 males) aged 18 to 35 years-old were included in the study. To identify exercise motives we used version translated of the Exercise Motivations Inventory (EMI-2). Males reported to give significantly more importance to exercise participation in terms of Physical Fitness and Competition, whereas females presented significantly higher ratings for Weight Management and Appearance. Exercise motives related to Disease Prevention, Weight Management and Health Rehabilitation significantly increased with increasing age. Motives exercise associated to Weight Management and Appearance were significantly higher in college students most economically privileged. In conclusion, these results will contribute to establish exercise promotion programs and to reduce exercise dropout rates among college students.
\end{abstract}

UnITERMS: Motivation; EMI-2; Physical activity; Young adult.

\title{
Resumen
}

Motivos para la práctica de ejercicio físico en universitarios y factores asociados

Objetivo de este estudio fue identificar los motivos de la práctica de ejercicio físico en universitarios según sexo, edad y situación económica. La muestra fue compuesta por 2.380 sujetos ( 1.213 chicas y 1167 chicos) de edades entre 18 y 35 años. Motivos para la práctica de ejercicio físico fueron identificados por la versión traducida del Exercise Motivations Inventory (EMI-2). Los chicos apuntaron ofrecer significativamente mayor importancia para la práctica de ejercicio físico por razones identificadas con Aptitud Física y Competición, y chicas con Control del Peso Corporal y Apariencia Física. Exposición de motivos relacionados con la Prevención de Enfermedades, Control del Peso Corporal y Rehabilitación de la Salud aumentó significativamente con la edad. Motivos asociados con el Control del Peso Corporal y Apariencia Física presentaron importancia significativamente mayor por los universitarios más privilegiados económicamente. En conclusión, resultados encontrados pueden contribuir a diseñar programas para promover el ejercicio físico y la reducción de posibles casos de abandono entre los universitarios.

Palabras clave: Motivación; EMI-2; Actividad física; Adulto joven.

\section{Referências}

ANDERSON, C.B. When more is better: number of motives and reasons for quitting as correlates of physical activity in women. Health Education Research, Oxford, v.18, n.5, p.525-37, 2003.

ANDRADE BASTOS, A.; SALGUERO A.; GONZALES-BOTO, R.; MARQUEZ, S. Motives for participation in physical activity by Brazilian adults. Perceptual and Motor Skills, Missoula, v.102, n.2, p.358-67, 2006.

ASSOCIAÇÃO BRASILEIRA DE EMPRESAS DE PESQUISA (ABEP). Critério padrão de classificação econômica Brasil/2008. São Paulo: ABEP. 2008.

BAUMAN, A.E. Updating the evidence that physical activity is good for health: an epidemiological review 2000-2003. Journal of Science and Medicine in Sport, Sydney, v.7, p.6-19, ano?. Supplement1.

BRUNET, J.; SABISTON, C.N. Exploring motivations for physical activity across the adult lifespan. Psychology of Sport and Exercise, Oxford, v.12, n.2, p.99-105, 2011.

CAGAS, J.Y.; TORRE, B.; MANALASTAS, E.J. Why do Filipinos exercise? Exploring motives from the perspective of Filipinos youth. In: CHIA, M.; WANG, J.; BALASEKARAN, G.; CHATZISARANTIS, N. (Eds.). Proceedings of the III International Conference of Physical Education and Sports Science. Singapore: National Institute of Education. 2010. p.243-48. 
CALFAS, K.J.; SALLIS, J.F.; LOVATO, C.Y.; CAMPBELL, J. Physical activity and its determinants before and after college graduation. Medicine, Exercise, Nutrition and Health, Cambridge, v.3, p.323-34, 1994.

CAPDEVILA, L.; NIÑEROLA, J.; PINTANEL, M. Motivation and physical activity: the self-report of motives for the practice of exercising. Revista de Psicologia del Deporte, Barcelona, v.13, n.1, p.55-74, 2004.

CASH, T.F.; NOVY, P.L.; GRANT, J.R. Why do women exercise? Factor analysis and further validation of the reasons for the exercise inventory. Perceptual and Motor Skills, Missoula, v.78, p.539-44, 2004.

CHEN, W. Chinese and American college students' motives for participation in physical activities. Perceptual and Motor Skills, Missoula, v.87, n.3Pt2, p.1463-70, 1998.

DACEY, M.; BALTZELL, A.; ZAICHKOWSKY, L. Older adults' intrinsic and extrinsic motivation toward physical activity. American Journal of Health Behavior, Oak Ridge, v.32, n.6, p.570-82, 2008.

DECI, E.L.; RYAN, R.M. Intrinsic motivacion and self-determination in human behavior. New York: Plenum, 1985. EBBEN, W.; BRUDZYNSKI, L. Motivations and barriers to exercise among college students. Journal of Exercise Physiology, Duluth, v.11, n.5, p.1-11, 2008.

GUEDES, D.P.; LEGNANI, R.F.S.; LEGNANI, E. Propriedades psicométricas da versão brasileira do Exercise Motivations Inventory (EMI-2). Motriz: Revista de Educação Física, Rio Claro, 2011. No prelo.

HAGGER, M.S.; CHATZISARANTIS, N.L.D. Self-determination theory and the psychology of exercise. International Review of Sport and Exercise Psychology, London, v.2, n.1. p.79-103, 2008.

HASKELL, W.L.; LEE, I-M; PATE, R.R.; POWELL, K.E.; BLAIR, S.N.; FRANKLIN, B.A.; MACERA, C.A.; HEATH, G.W.; THOMPSON, P.D.; BAUMAN, A. Physical activity and public health: updated recommendation for adults from the American College of Sports Medicine and the American Heart Association. Circulation, Dallas, v.116, p.1081-93, 2007. INGLEDEW, D.K.; MARKLAND, D. The role of motives in exercise participation. Psychology and Health, London, v.23, n.7, p.807-28, 2008.

INGLEDEW, D.K.; MARKLAND, D.; FERGUSON, E. Three levels of exercise motivations. Applied Psychology: Health and Well-Being, London, v.1, n.3, p.336-55, 2009.

INGLEDEW, D.K.; SULLIVAN, G. Effects of body mass and body image on exercise motives in adolescence. Psychology of Sport and Exercise, Oxford, v.3, p.323-38, 2002.

KEATING, X.D.; GUAN, J.; PIÑERO, J.C.; BRIDGES, D.M. A meta-analysis of college students' physical activity behaviors. Journal of American College Health, Hanover, v.54, n.2, p.116-25, 2005.

KEELE R. Development of the exercise motivation questionnaire with Mexican American adults. Journal of Nursing Measurement, New York, v.17, n.3, p.183-94, 2009.

KILPATRICK, M.; HEBERT, E.; BARTHOLOMEW, J. College students' motivation for physical activity: differentiating men's and women's motives for sport participation and exercise. Journal of American College Health, Hanover, v.54, n.2, p.87-94, 2005.

LOZE, G.M.; COLLINS, D.J. Muscular development motives for exercise participation: the missing variable in current questionnaire analysis? Journal of Sports Sciences, London, v.16, n.8, p.761-67, 1998.

MALTBY, J.; DAY, L. The relationship between exercise motives and psychological well-being. Journal of Psychology, Philadelphia, v.135, n.6, p.651-660, 2001.

MARKLAND, D.; INGLEDEW, D.K. The measurement of exercise motives: factorial validity and invariance across gender of a revised Exercise Motivations Inventory. British Journal of Health Psychology, London, v.2, p.361-76, 1997. NETZ, Y.; RAVIV, S. Age differences in motivational orientation toward physical activity: an application of social cognitive theory. Journal of Psychology, Philadelphia, v.138, p.35-48, 2004.

NOCON, M.; HIEMANN, T.; MÜLLER-RIEMENSCHNEIDER, F.; THALAU, F.; ROLL, D.; WILLICH, S.N. Association of physical activity with all-cause and cardiovascular mortality: a systematic review and meta-analysis. European Journal of Cardiovascular Prevention \& Rehabilitation, Sophia Antipolis, v.15, n.3, p.239-46, 2008.

QUINDRY, J.C.; YOUNT, D.; O’BRYANT, H.; RUDISILL, M.E. Exercise engagement is differentially motivated by age-dependent factors. American Journal of Health Behavior, Oak Ridge, v.35, n.3, p.334-45, 2011.

VIERLING, K.K.; STANDAGE, M.; TREASURE, D.C. Predicting attitudes and physical activity in an at-risk minority youth sample: a test of self-determination theory. Psychology of Sport and Exercise, Oxford, v.8, p.795-817, 2007.

WARBURTON, D.E.R.; NICOL, C.W.; BREDIN, S.S.D. Health benefits of physical activity: the evidence. Canadian Medical Association Journal, Ottawa, v.174, p.801-09, 2006.

WEINBERG, R.; GOULD, D. Foundations of sport and exercise psychology. 5th ed. Champaign: Human Kinetics, 2011.

688 • Rev. bras. Educ. Fís. Esporte, São Paulo, v.26, n.4, p.679-89, out./dez. 2012 
WILSON, P.M.; RODGERS, W.M. The relationship between exercise motives and physical self-esteem in female exercise participants: an application of self-determination theory. Journal of Applied Biobehavioral Research, Malden, v.7, p.30-43, 2002.

WITHALL, J.; JAGO, R.; FOX, K.R. Why some do but most don't. Barriers and enablers to engaging low-income groups in physical activity programmes: a mixed methods study. BMC Public Health, London, v.11, p.507, 2011.

WORLD HEALTH ORGANIZATION (WHO). Global recommendations on physical activity for health. Geneva: WHO, 2010.

YAN, J.H.; McCULLAGH, P.J. Cultural influence on youth's motivation of participation in physical activity. Journal of Sport Behavior, New York, v.27, p.378-90, 2004.

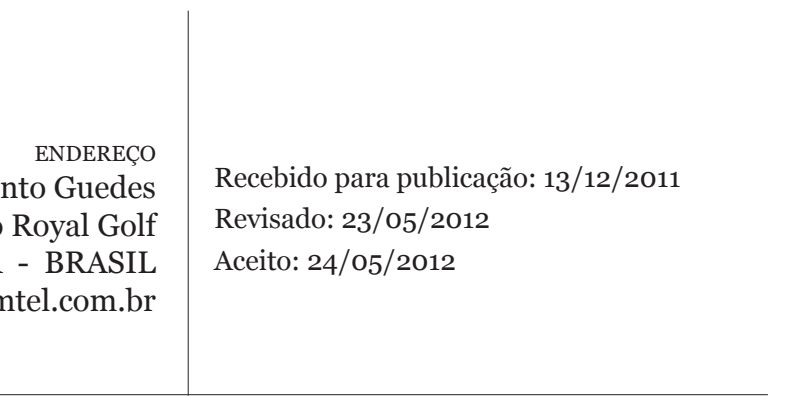

